\title{
Meat consumption and risk of 25 common conditions: outcome-wide analyses in 475,000 men and women in the UK Biobank study
}

Keren Papier ${ }^{1 *}$ (D, Georgina K. Fensom¹,2, Anika Knuppel ${ }^{1}$, Paul N. Appleby ${ }^{1}$, Tammy Y. N. Tong ${ }^{1}$, Julie A. Schmidt', Ruth C. Travis ${ }^{1}$, Timothy J. Key ${ }^{1}$ and Aurora Perez-Cornago ${ }^{1}$

\begin{abstract}
Background: There is limited prospective evidence on the association between meat consumption and many common, non-cancerous health outcomes. We examined associations of meat intake with risk of 25 common conditions (other than cancer).
\end{abstract}

Methods: We used data from 474,985 middle-aged adults recruited into the UK Biobank study between 2006 and 2010 and followed up until 2017 (mean follow-up 8.0 years) with available information on meat intake at baseline (collected via touchscreen questionnaire), and linked hospital admissions and mortality data. For a large sub-sample $(\sim 69,000)$, dietary intakes were re-measured three or more times using an online, 24-h recall questionnaire.

Results: On average, participants who reported consuming meat regularly (three or more times per week) had more adverse health behaviours and characteristics than participants who consumed meat less regularly, and most of the positive associations observed for meat consumption and health risks were substantially attenuated after adjustment for body mass index (BMI). In multi-variable adjusted (including BMI) Cox regression models corrected for multiple testing, higher consumption of unprocessed red and processed meat combined was associated with higher risks of ischaemic heart disease (hazard ratio (HRs) per $70 \mathrm{~g} /$ day higher intake 1.15, 95\% confidence intervals (Cls) 1.07-1.23), pneumonia (1.31, 1.18-1.44), diverticular disease (1.19, 1.11-1.28), colon polyps (1.10, 1.06-1.15), and diabetes $(1.30,1.20-1.42)$; results were similar for unprocessed red meat and processed meat intakes separately. Higher consumption of unprocessed red meat alone was associated with a lower risk of iron deficiency anaemia (IDA: HR per $50 \mathrm{~g} /$ day higher intake $0.80,95 \% \mathrm{Cls} 0.72-0.90$ ). Higher poultry meat intake was associated with higher risks of gastro-oesophageal reflux disease (HR per $30 \mathrm{~g} /$ day higher intake 1.17, 95\% Cls 1.09-1.26), gastritis and duodenitis (1.12, 1.05-1.18), diverticular disease (1.10, 1.04-1.17), gallbladder disease (1.11, 1.04-1.19), and diabetes $(1.14,1.07-1.21)$, and a lower IDA risk (0.83, 0.76-0.90).

(Continued on next page)

\footnotetext{
* Correspondence: Keren.Papier@ndph.ox.ac.uk

'Cancer Epidemiology Unit, Nuffield Department of Population Health, University of Oxford, Richard Doll Building, Old Road Campus, Oxford OX3 7LF, UK

Full list of author information is available at the end of the article
}

C C The Author(s). 2021 Open Access This article is licensed under a Creative Commons Attribution 4.0 International License, which permits use, sharing, adaptation, distribution and reproduction in any medium or format, as long as you give appropriate credit to the original author(s) and the source, provide a link to the Creative Commons licence, and indicate if changes were made. The images or other third party material in this article are included in the article's Creative Commons licence, unless indicated otherwise in a credit line to the material. If material is not included in the article's Creative Commons licence and your intended use is not permitted by statutory regulation or exceeds the permitted use, you will need to obtain permission directly from the copyright holder. To view a copy of this licence, visit http://creativecommons.org/licenses/by/4.0/ The Creative Commons Public Domain Dedication waiver (http://creativecommons.org/publicdomain/zero/1.0/) applies to the data made available in this article, unless otherwise stated in a credit line to the data. 
(Continued from previous page)

Conclusions: Higher unprocessed red meat, processed meat, and poultry meat consumption was associated with higher risks of several common conditions; higher BMI accounted for a substantial proportion of these increased risks suggesting that residual confounding or mediation by adiposity might account for some of these remaining associations. Higher unprocessed red meat and poultry meat consumption was associated with lower IDA risk.

Keywords: Red meat, Processed meat, Poultry, Prospective cohort study, UK Biobank, Risk, Outcome-wide

\section{Background}

The World Health Organization [1] and many national dietary advice bodies (e.g. the UK dietary guidelines [2]) have in recent years recommended a reduction of red and processed meat consumption, based on consistent evidence linking high processed meat, and probably red meat consumption, with colorectal cancer risk [1]. While the association between meat intake and cancer risk has been comprehensively studied [3, 4], there is less information on the association between meat consumption, especially poultry meat, and incidence of major noncancerous health outcomes [5]. Although several prospective studies have assessed the association of unprocessed red meat and processed meat consumption with risk of cardiovascular disease [6] and diabetes [7], the evidence is equivocal for ischaemic heart disease [8-10] and limited for stroke subtypes (e.g. haemorrhagic stroke [11]). Moreover, the evidence on poultry and CVD is particularly limited [12], while the evidence on poultry and diabetes is unclear $[13,14]$. This lack of clear and available evidence for major non-cancerous health outcomes might relate to outcome selection bias (i.e. only reporting the outcomes that are found to be statistically significant [15]), differences in the definition of outcomes and exposures, sample size, control of confounders, and/or length of follow-up used among different studies. Examining the association between meat consumption and multiple noncancerous health outcomes in the same large cohort may help to clarify these associations [16].

This study uses an outcome-wide approach to prospectively examine associations of meat consumption with risk of 25 common conditions identified as the 25 leading causes of hospital admission (other than cancer) in a large UK cohort.

\section{Methods}

\section{Study population}

We used data from the UK Biobank study, a cohort of 503,317 men and women from across the UK [17]. Potential participants were recruited through the $\mathrm{Na}$ tional Health Service (NHS) Patient Registers and invited to attend one of the 22 assessment centres between 2006 and 2010. Participants joining the study completed a baseline touchscreen questionnaire, provided anthropometric and biological data, and gave informed consent for their health to be followed up through linkage to electronic medical records.

\section{Assessment of dietary intake}

Dietary intake was assessed using a touchscreen dietary questionnaire administered to all participants at baseline that included 29 questions on diet, assessing the consumption frequency of each listed food. Responses to the five questions on meat (unprocessed beef, unprocessed lamb/mutton, unprocessed pork, unprocessed poultry, and processed meat) were assigned values for frequency per week (never $=0$, less than once per week $=$ 0.5 , once per week $=1,2-4$ times per week $=3,5-6$ times per week $=5.5$, and once or more a day $=7$ ). We then collapsed these meat intake frequencies into three or four categories to create approximately equal-sized groups (see Additional file 1: Methods 1 for additional detail).

Participants recruited after 2009, as well as participants who provided UK Biobank with an email address and agreed to be re-contacted, were additionally invited to complete the Oxford WebQ [18], an online 24-h recall questionnaire. Participants were asked to select how many portions of each food item they consumed over the previous $24 \mathrm{~h}$, enabling calculation of mean grams per day by multiplying frequencies of consumption by standard portion sizes. Similar foods were then grouped together into meat types to match the touchscreen dietary questionnaire. We then assigned the mean WebQ meat intakes in participants who had completed at least three WebQs to each touchscreen meat category defined for all participants. Using these assigned means, we calculated trends in risk across categories of baseline meat intakes $[4,19]$. This approach uses repeat measurements to estimate usual mean meat intakes in each category of meat intake, thereby reducing random error in the assessment of usual meat consumption (see Additional file 1: Methods 1 for additional detail).

\section{Assessment of health outcomes}

The outcomes of interest in this study were incident cases of 25 common conditions. The conditions selected were those identified as the 25 leading, well-defined causes of non-cancerous hospital admission in this cohort based on the primary International Classification of 
Diseases (ICD) 10 diagnosis codes recorded during admission. Some of the commonest causes of hospital admission in this cohort (e.g. nausea or heartburn) were not considered to be separate conditions, because they were not well-defined and/or were likely to be associated with a diverse range of underlying conditions. Moreover, although diabetes was not among the 25 most common primary diagnoses associated with admission, it is a common secondary reason for admission and therefore any diagnosis of diabetes was included among the 25 common conditions examined (see Additional file 1: Table 1 for selected conditions and relevant diagnosis, and procedure codes).

Participant information on cause-specific in-patient hospital admissions and deaths (primary cause for all outcomes except diabetes which also included any diagnosis for hospital admission or mention on the death certificate) was obtained through linkage to the NHS Central Registers. For participants in England, Hospital Episode Statistics (HES) and information on date and cause of death were available until the 31st of March 2017; for participants in Scotland, Scottish Morbidity Records and information on date and cause of death were available until the 31st of October 2016; and for participants in Wales, the Patient Episode Database and information on date and cause of death were available until the 29th of February 2016. We also obtained information on cancer registrations (including date and cancer site) from the NHS Central Registers (see Additional file 1: Methods 2 and Additional file 1: Table 1 for information on exclusion, diagnosis and procedure codes).

\section{Exclusions}

Of the 503,317 recruited participants, 28,332 were excluded due to study withdrawals, prevalent cancer (except nonmelanoma skin cancer, ICD-10 C44), or because their genetic sex differed from their reported gender, resulting in a maximal study sample of 474,985 (94\%). Participants with a relevant diagnosis or procedure prior to recruitment, ascertained through the touchscreen questionnaire, nurseguided interviews, and hospital admission data, were excluded for each condition (see Additional file 1: Table 1 for details about the exclusions for each outcome). Participants who did not report their meat intake in the touchscreen questionnaire or reported 'prefer not to say' or 'do not know' were classified as missing and excluded for the respective exposure analyses (see Additional file 1: Fig. 1 for participant flowchart and Additional file 1: Tables 6, 7, 8, 9, and 10 for total numbers for each exposure and outcome).

\section{Statistical analysis}

We used Cox proportional hazards regression models to assess associations between meat consumption and risk for incident cases separately for each disease or condition, calculating trends using the mean meat intakes calculated using the WebQ questionnaires for each category from the touchscreen questionnaire and the trend test variables. Participants' survival time in personyears was calculated from their age at recruitment until their age at hospital admission, death, loss to follow-up, or administrative censoring. All analyses were stratified by sex, age at recruitment, and geographical region (Model 0). In Model 1, we estimated hazard ratios (HRs) and 95\% confidence intervals (CIs) adjusted for race, Townsend deprivation index [20], education, employment, smoking, alcohol consumption, and physical activity, and in women, we additionally adjusted for menopausal status, hormone replacement therapy, oral contraceptive pill use, and parity. In Model 2, we further adjusted for total fruit and vegetable intake, cereal fibre intake score (calculated by multiplying the frequency of consumption of bread and breakfast cereal by the fibre content of these foods [21]), oily fish intake, and non-oily fish intake. For Model 3, we added adjustment for body mass index (BMI). Missing data for all covariates was minimal $(<10 \%)$ and thus a 'missing' category was created for each covariate (see Figs. 1, 2, 3, and 4 footnotes and Additional file 1: Methods 3 for full adjustment description with definitions of categories)

\section{Sensitivity analyses}

To examine whether the associations between meat intake and risk of incidence for specific diagnoses could be affected by reverse causality or residual confounding by smoking, we repeated the analyses (1) after excluding the first 4 years of follow-up and (2) restricted to never smokers.

All analyses were conducted using STATA version 15.1 (Stata Corp LP, College Station, TX). All $P$ values were two-sided and Bonferroni correction was used to allow for multiple testing (for 25 outcomes, $P<0.002$ ).

\section{Results \\ Baseline characteristics}

Table 1 shows baseline characteristics of participants by categories of unprocessed red meat and processed meat intake. Around one-third of participants consumed unprocessed red and/or processed meat once or more daily. On average, participants who consumed unprocessed red and processed meat regularly (three or more times per week) were more likely to be men, older, of White European race, retired, have higher BMI, smoke and consume alcohol, and consume less fruit and vegetables, fibre, and fish and more poultry meat; they were also less likely to have attained a tertiary education, and among women to have two or more children, not use oral contraceptives, use hormone replacement therapy, or be postmenopausal compared with participants who consumed meat less than three times per 
Table 1 Baseline characteristics of participants by unprocessed red and processed meat intake in UK Biobank $(n=467,741$, see Additional file 1: Fig. 1)

\begin{tabular}{|c|c|c|c|c|}
\hline $\begin{array}{l}\text { Characteristic } \\
\text { Mean (SD) or } n(\%)\end{array}$ & $\begin{array}{l}0-1 \text { time/week } \\
N=44,019\end{array}$ & $\begin{array}{l}2 \text { times/week } \\
N=160,069\end{array}$ & $\begin{array}{l}3-4 \text { times/week } \\
N=140,674\end{array}$ & $\begin{array}{l}\geq 5 \text { times } / \text { week } \\
N=122,979\end{array}$ \\
\hline \multicolumn{5}{|l|}{ Sociodemographic } \\
\hline \multicolumn{5}{|l|}{ Sex, $n(\%)$} \\
\hline Women & $31,318(71.1)$ & $101,747(63.6)$ & $71,679(51.0)$ & $47,657(38.8)$ \\
\hline Men & $12,701(28.9)$ & $58,322(36.4)$ & $68,995(49.0)$ & $75,322(61.2)$ \\
\hline Age (years), mean (SD) & $54.9(8.2)$ & $56.5(8.0)$ & $56.4(8.1)$ & $56.5(8.2)$ \\
\hline \multicolumn{5}{|l|}{ Race, $n(\%)$} \\
\hline White & $38,451(87.4)$ & $151,727(94.8)$ & $134,509(95.6)$ & $116,815(95.0)$ \\
\hline Asian or Asian British & $3468(7.9)$ & $2914(1.8)$ & $2245(1.6)$ & $1897(1.5)$ \\
\hline Black or Black British & $886(2.0)$ & $2607(1.6)$ & $1685(1.2)$ & $2047(1.7)$ \\
\hline Mixed race/others & $997(2.3)$ & $2323(1.5)$ & $1807(1.3)$ & $1774(1.4)$ \\
\hline Unknown & $217(0.5)$ & $498(0.3)$ & $428(0.3)$ & $446(0.4)$ \\
\hline \multicolumn{5}{|l|}{ Townsend deprivation, $n$ (\%) } \\
\hline Most affluent (mean - 4.7) & $7036(16.0)$ & $32,846(20.5)$ & $29,803(21.2)$ & $24,666(20.1)$ \\
\hline 2 (mean - 3.3) & $7524(17.1)$ & $32,745(20.5)$ & $29,079(20.7)$ & 24,304 (19.8) \\
\hline $3($ mean -2.1$)$ & $8341(18.9)$ & $32,737(20.5)$ & $28,324(20.1)$ & $24,343(19.8)$ \\
\hline $4($ mean -0.1$)$ & $10,146(23.0)$ & $31,753(19.8)$ & $27,446(19.5)$ & $24,066(19.6)$ \\
\hline Most deprived (mean 3.8) & $10,910(24.8)$ & $29,773(18.6)$ & $25,869(18.4)$ & $25,448(20.7)$ \\
\hline Unknown & $62(0.1)$ & $215(0.1)$ & $153(0.1)$ & $152(0.1)$ \\
\hline \multicolumn{5}{|l|}{ Qualification, $n(\%)$} \\
\hline College/university degree/NVQ & $28,490(64.7)$ & $96,075(60.0)$ & $82,364(58.5)$ & $72,437(58.9)$ \\
\hline National examination at ages $17-18$ & $2491(5.7)$ & $8708(5.4)$ & $7795(5.5)$ & $6626(5.4)$ \\
\hline National examination at age 16 & $6321(14.4)$ & $27,682(17.3)$ & $24,133(17.2)$ & $19,675(16.0)$ \\
\hline Others/unknown & $6717(15.3)$ & $27,604(17.2)$ & $26,382(18.8)$ & 24,241 (19.7) \\
\hline \multicolumn{5}{|l|}{ Employment, $n(\%)$} \\
\hline In paid employment & $27,650(62.8)$ & $93,965(58.7)$ & $81,641(58.0)$ & $70,102(57.0)$ \\
\hline Pension & $10,229(23.2)$ & $48,219(30.1)$ & $42,698(30.4)$ & $37,181(30.2)$ \\
\hline Not in paid employment & $5556(12.6)$ & $16,487(10.3)$ & $15,236(10.8)$ & $14,560(11.8)$ \\
\hline Unknown & $584(1.3)$ & $1398(0.9)$ & $1099(0.8)$ & $1136(0.9)$ \\
\hline \multicolumn{5}{|l|}{ Physical measurements } \\
\hline BMI $\left(\mathrm{kg} / \mathrm{m}^{2}\right)$, mean $(\mathrm{SD})$ & $25.9(4.7)$ & $27.1(4.7)$ & $27.6(4.8)$ & $28.1(4.9)$ \\
\hline \multicolumn{5}{|l|}{ Lifestyle } \\
\hline \multicolumn{5}{|l|}{ Smoking, $n(\%)$} \\
\hline Never & $26,347(59.9)$ & $90,448(56.5)$ & $76,682(54.5)$ & $62,688(51.0)$ \\
\hline Former & $13,962(31.7)$ & $54,796(34.2)$ & $48,534(34.5)$ & $43,268(35.2)$ \\
\hline Current $<15$ cigarettes/day & $1297(2.9)$ & $4581(2.9)$ & $4247(3.0)$ & $4074(3.3)$ \\
\hline Current $\geq 15$ cigarettes/day & $1030(2.3)$ & $4825(3.0)$ & $6015(4.3)$ & $7622(6.2)$ \\
\hline Current, amount unknown & $1213(2.8)$ & $4876(3.0)$ & $4727(3.4)$ & $4914(4.0)$ \\
\hline Unknown & $170(0.4)$ & $543(0.3)$ & $469(0.3)$ & $413(0.3)$ \\
\hline \multicolumn{5}{|l|}{ Physical activity level, $n$ (\%) } \\
\hline Low $<10$ excess METs & $12,405(28.2)$ & $49,709(31.1)$ & $45,467(32.3)$ & $39,706(32.3)$ \\
\hline Moderate 10 to $<50$ excess METs & $22,380(50.8)$ & $80,039(50.0)$ & $68,196(48.5)$ & $58,006(47.2)$ \\
\hline High $\geq 50$ excess METs & $7769(17.6)$ & $24,715(15.4)$ & $21,860(15.5)$ & 20,458 (16.6) \\
\hline
\end{tabular}


Table 1 Baseline characteristics of participants by unprocessed red and processed meat intake in UK Biobank $(n=467,741$, see Additional file 1: Fig. 1) (Continued)

\begin{tabular}{|c|c|c|c|c|}
\hline $\begin{array}{l}\text { Characteristic } \\
\text { Mean (SD) or } n(\%)\end{array}$ & $\begin{array}{l}0-1 \text { time/week } \\
N=44,019\end{array}$ & $\begin{array}{l}2 \text { times/week } \\
N=160,069\end{array}$ & $\begin{array}{l}3-4 \text { times/week } \\
N=140,674\end{array}$ & $\begin{array}{l}\geq 5 \text { times/week } \\
N=122,979\end{array}$ \\
\hline Unknown & $1465(3.3)$ & $5606(3.5)$ & $5151(3.7)$ & 4809 (3.9) \\
\hline \multicolumn{5}{|l|}{ Alcohol intake, $n(\%)$} \\
\hline Non-drinkers & $7503(17.0)$ & $11,938(7.5)$ & $9611(6.8)$ & $7790(6.3)$ \\
\hline$<1$ g/day & $6814(15.5)$ & $19,866(12.4)$ & $14,469(10.3)$ & $10,861(8.8)$ \\
\hline 1 to $<10 \mathrm{~g} /$ day & $14,742(33.5)$ & $57,211(35.7)$ & $43,226(30.7)$ & $31,588(25.7)$ \\
\hline 10 to $<20 \mathrm{~g} /$ day & $7984(18.1)$ & $36,199(22.6)$ & $31,681(22.5)$ & $25,627(20.8)$ \\
\hline $20+$ g/day & $6740(15.3)$ & $34,045(21.3)$ & $41,068(29.2)$ & $46,544(37.8)$ \\
\hline Unknown & $236(0.5)$ & $810(0.5)$ & $619(0.4)$ & $569(0.5)$ \\
\hline \multicolumn{5}{|l|}{ Diet } \\
\hline Fruit and vegetable intake (s/day), mean (SD) & $5.59(3.19)$ & $4.89(2.54)$ & $4.50(2.45)$ & $4.33(2.50)$ \\
\hline Cereal fibre intake (g/day), mean (SD) & $4.66(3.11)$ & $4.52(2.89)$ & $4.52(2.91)$ & $4.44(2.96)$ \\
\hline \multicolumn{5}{|l|}{ Oily fish, $n(\%)$} \\
\hline 0 time/week & $12,568(28.6)$ & $12,192(7.6)$ & $13,264(9.4)$ & 13,296 (10.8) \\
\hline$<1$ time/week & $8444(19.2)$ & $52,209(32.6)$ & $49,864(35.4)$ & $44,506(36.2)$ \\
\hline 1 time/week & $11,522(26.2)$ & $62,503(39.0)$ & $55,502(39.5)$ & $46,265(37.6)$ \\
\hline$\geq 2$ times/week & $11,299(25.7)$ & $32,572(20.3)$ & $21,480(15.3)$ & $18,279(14.9)$ \\
\hline Unknown & $186(0.4)$ & $593(0.4)$ & $564(0.4)$ & $633(0.5)$ \\
\hline \multicolumn{5}{|l|}{ Non-oily fish, $n$ (\%) } \\
\hline$<1$ time/week & $19,962(45.3)$ & $54,020(33.7)$ & $45,185(32.1)$ & $38,703(31.5)$ \\
\hline 1 time/week & $14,427(32.8)$ & $79,177(49.5)$ & $74,260(52.8)$ & $63,929(52.0)$ \\
\hline$\geq 2$ times/week & $9432(21.4)$ & $26,393(16.5)$ & 20,801 (14.8) & $19,887(16.2)$ \\
\hline Unknown & $198(0.4)$ & $479(0.3)$ & $428(0.3)$ & $460(0.4)$ \\
\hline \multicolumn{5}{|l|}{ Poultry meat, $n(\%)$} \\
\hline 0-1 time/week & $26,359(59.9)$ & $23,487(14.7)$ & $13,477(9.6)$ & $10,760(8.7)$ \\
\hline 2 times/week & $7141(16.2)$ & $61,114(38.2)$ & $56,696(40.3)$ & $42,291(34.4)$ \\
\hline$\geq 3$ times/week & $10,461(23.8)$ & $75,358(47.1)$ & $70,405(50.0)$ & 69,835 (56.8) \\
\hline Unknown & $58(0.1)$ & $110(0.1)$ & $96(0.1)$ & $93(0.1)$ \\
\hline \multicolumn{5}{|l|}{ Women factors } \\
\hline \multicolumn{5}{|l|}{ Menopausal status, n (\%) } \\
\hline Premenopausal & $8960(28.6)$ & $22,946(22.6)$ & $17,038(23.8)$ & $11,207(23.5)$ \\
\hline Postmenopausal & $20,588(65.7)$ & $73,267(72.0)$ & $50,660(70.7)$ & $33,725(70.8)$ \\
\hline Unknown & $1770(5.7)$ & $5534(5.4)$ & 3981 (5.6) & $2725(5.7)$ \\
\hline \multicolumn{5}{|l|}{ Parity, n (\%) } \\
\hline 0 births & 8314 (26.5) & 19,781 (19.4) & $11,737(16.4)$ & 7298 (15.3) \\
\hline $1-2$ births & $16,231(51.8)$ & $58,204(57.2)$ & $42,076(58.7)$ & $27,654(58.0)$ \\
\hline$\geq 3$ births & 6717 (21.4) & $23,671(23.3)$ & $17,812(24.8)$ & 12,655 (26.6) \\
\hline Unknown & $56(0.2)$ & $91(0.1)$ & $54(0.1)$ & $50(0.1)$ \\
\hline \multicolumn{5}{|l|}{ HRT use, $n(\%)$} \\
\hline Never & $21,436(68.4)$ & $61,911(60.8)$ & $44,006(61.4)$ & 28,975 (60.8) \\
\hline Past & 7951 (25.4) & $33,162(32.6)$ & $23,018(32.1)$ & $15,560(32.6)$ \\
\hline Current & $1773(5.7)$ & $6379(6.3)$ & $4429(6.2)$ & $2897(6.1)$ \\
\hline Unknown & $158(0.5)$ & $295(0.3)$ & $226(0.3)$ & $225(0.5)$ \\
\hline
\end{tabular}


Table 1 Baseline characteristics of participants by unprocessed red and processed meat intake in UK Biobank $(n=467,741$, see Additional file 1: Fig. 1) (Continued)

\begin{tabular}{|c|c|c|c|c|}
\hline Characteristic & 0-1 time/week & 2 times/week & 3-4 times/week & $\geq 5$ times/week \\
\hline Mean (SD) or $n(\%)$ & $N=44,019$ & $N=160,069$ & $N=140,674$ & $N=122,979$ \\
\hline \multicolumn{5}{|l|}{ OCP use, $n(\%)$} \\
\hline Never & $6596(21.1)$ & 18,180 (17.9) & $12,849(17.9)$ & 8917 (18.7) \\
\hline Past & 23,874 (76.2) & $81,528(80.1)$ & $57,266(79.9)$ & $37,642(79.0)$ \\
\hline Current & $691(2.2)$ & $1812(1.8)$ & $1388(1.9)$ & 918 (1.9) \\
\hline Unknown & $157(0.5)$ & $227(0.2)$ & $176(0.2)$ & $180(0.4)$ \\
\hline
\end{tabular}

The $x^{2}$ test was used to compare the distribution between meat intakes for all categorical variables. Analysis of variance (ANOVA) was used to compare the means between meat intakes. The $P$ heterogeneity between meat intakes was $<0.001$ for all variables. All dietary data come from the touchscreen questionnaire. $B M I$ body mass index, HRT hormone replacement therapy, OCP oral contraceptive pill use, NVQ national vocational qualification, s/day servings/day, $g / d a y$ grams/day

week $(P<0.001$ for heterogeneity between meat intakes for all baseline characteristics). Participants who consumed higher amounts of unprocessed red meat were more likely to consume higher amounts of processed meat and poultry meat (see Additional file 1: Table 3). Baseline characteristics in relation to poultry meat consumption were somewhat different (see Additional file 1: Table 5).

\section{Risk analyses}

Figures 1, 2, 3, and 4 present the numbers of incident cases for 25 common conditions and their HRs and 95\% CIs per unit higher intake of meat for the multipleadjusted model (Model 3) over an average follow-up of 8.0 years (standard deviation 1.0 ). Risks by categories of meat intake at baseline for Models $0-3$ can be found in Additional file 1: Tables 6, 7, 8, 9, and 10. Overall, many of the positive associations were substantially attenuated, and in some cases were no longer statistically significant, with the additional adjustment for BMI (Model 3). Here we describe the results for Model 3 that were robust to correction for multiple testing. Risks for total meat intake (unprocessed red, processed, and poultry meat combined) did not yield any additional associations and these results are therefore only presented in Additional file 1: Table 6 and Fig. 7.

\section{Total unprocessed red meat and processed meat}

Total unprocessed red meat and processed meat intake was associated with a higher risk of ischaemic heart disease (IHD) (HR per $70 \mathrm{~g} /$ day higher intake $=1.15$, 95\% CI 1.07-1.23), pneumonia (1.31, 1.18-1.44), diverticular disease $(1.19,1.11-1.28)$, colon polyps (1.10, $1.06-1.15)$, and diabetes $(1.30,1.20-1.42)$ (Fig. 1).

\section{Unprocessed red meat}

Unprocessed red meat intake was associated with a higher risk of IHD (HR per $50 \mathrm{~g} /$ day higher intake = 1.16, 95\% CI 1.08-1.25), pneumonia (1.22, 1.10-1.35), diverticular disease $(1.17,1.09-1.26)$, colon polyps (1.08,
1.04-1.13), and diabetes $(1.21,1.11-1.32)$, and a lower risk of IDA (0.80, 0.72-0.90) (Fig. 2).

\section{Processed meat}

Processed meat intake was associated with a higher risk of IHD (HR per $20 \mathrm{~g}$ /day higher intake $=1.09,95 \% \mathrm{CI}$ 1.04-1.15), pneumonia (1.23, 95\% CI 1.15-1.32), diverticular disease $(1.11,1.06-1.17)$ colon polyps $(1.08,95 \%$ CI 1.05-1.11), and diabetes (1.24, 1.17-1.32) (Fig. 3).

\section{Poultry meat}

Poultry meat intake was associated with a higher risk of gastro-oesophageal reflux disease (GERD) (HR per $30 \mathrm{~g} /$ day higher intake $=1.17,95 \%$ CI 1.09-1.26), gastritis and duodenitis (1.12, 1.05-1.18), diverticular disease (1.10, 1.04-1.17), gallbladder disease (1.11, 1.04-1.19), and diabetes $(1.14,1.07-1.21)$, and a lower risk of IDA (0.83, 0.76-0.90) (Fig. 4).

\section{Sensitivity analysis}

Associations were similar when excluding the first 4 years of follow-up and in never smokers (Additional file 1: Figs. 2, 3, 4,5 , and 6.). However, we did note a positive association between unprocessed red and processed meat intake (combined) and haemorrhagic stroke (HR per $70 \mathrm{~g} /$ day higher intake $=1.53,95 \%$ CI 1.10-2.14) in participants diagnosed after 4 or more years of follow-up and that the associations between unprocessed red meat intake and diabetes risk, and processed meat intake and IHD risk, were no longer statistically significant in never smokers.

\section{Discussion}

In this large, prospective cohort of nearly 0.5 million UK adults, we observed that after allowing for multiple testing, higher consumption of unprocessed red and processed meat combined was associated with higher risks of IHD, pneumonia, diverticular disease, colon polyps, and diabetes, and higher consumption of poultry meat was associated with higher risks of GERD, gastritis and duodenitis, diverticular disease, gallbladder disease, and diabetes. Differences in BMI across the categories of meat 


\begin{tabular}{|c|c|c|c|c|c|c|}
\hline Disease subgroup & Total $\mathbf{N}$ & Cases & & & $\mathrm{R}(95 \% \mathrm{Cl})$ per $70 \mathrm{~g} / \mathrm{d}$ & Ptrend \\
\hline \multicolumn{7}{|l|}{ Circulatory disease } \\
\hline Ischemic heart disease & 430714 & 13120 & & $\rightarrow-$ & $1.15(1.07,1.23)$ & $<0.001$ \\
\hline Atrial fibrillation and flutter & 430714 & 4741 & & & $0.89(0.79,0.99)$ & 0.040 \\
\hline Cerebrovascular disease & 430714 & 5330 & & $\longrightarrow-$ & $1.17(1.04,1.30)$ & 0.006 \\
\hline Ischaemic stroke & 430714 & 2338 & & 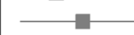 & $1.26(1.06,1.49)$ & 0.008 \\
\hline Haemorrhagic stroke & 430714 & 939 & & $=$ & $1.12(0.87,1.44)$ & 0.378 \\
\hline Venous thromboembolism & 457400 & 3751 & & $=$ & $1.07(0.94,1.22)$ & 0.303 \\
\hline Varicose veins & 445504 & 2633 & $=$ & & $0.94(0.81,1.09)$ & 0.391 \\
\hline Haemorrhoids & 448120 & 8296 & & & $1.07(0.99,1.17)$ & 0.098 \\
\hline \multicolumn{7}{|l|}{ Respiratory disease } \\
\hline Pneumonia & 459309 & 6350 & & $\rightarrow-$ & $1.31(1.18,1.44)$ & $<0.001$ \\
\hline \multicolumn{7}{|l|}{ Digestive disease } \\
\hline GERD & 439856 & 7010 & & & $0.97(0.89,1.07)$ & 0.566 \\
\hline Gastritis and duodenitis & 450997 & 11365 & & & $0.99(0.92,1.06)$ & 0.734 \\
\hline Inguinal hernia & 440760 & 8019 & & & $0.99(0.90,1.08)$ & 0.762 \\
\hline Noninfective enteritis and colitis & 453616 & 6235 & & $=-$ & $1.05(0.96,1.16)$ & 0.304 \\
\hline Diverticular disease & 455281 & 12816 & & $\rightarrow$ & $1.19(1.11,1.28)$ & $<0.001$ \\
\hline Colon polyps & 446048 & 37400 & & & $1.10(1.06,1.15)$ & $<0.001$ \\
\hline Gallbladder disease & 448382 & 8914 & & & $1.04(0.96,1.13)$ & 0.326 \\
\hline \multicolumn{7}{|l|}{ Joint disorder } \\
\hline Osteoarthritis & 417176 & 18080 & & & $0.97(0.92,1.03)$ & 0.311 \\
\hline \multicolumn{7}{|l|}{ Genitourinary disease } \\
\hline Kidney stones & 460559 & 2444 & & ב- & $1.02(0.87,1.19)$ & 0.797 \\
\hline Urinary tract infection & 459988 & 5205 & & & $1.07(0.96,1.19)$ & 0.219 \\
\hline Enlarged prostate & 205590 & 3539 & & & $1.01(0.88,1.16)$ & 0.893 \\
\hline Female genital prolapse & 239654 & 6062 & & - & $1.09(0.99,1.20)$ & 0.074 \\
\hline \multicolumn{7}{|l|}{ Other diseases } \\
\hline Uterine fibroids & 181786 & 9258 & & & $0.99(0.92,1.06)$ & 0.776 \\
\hline Iron deficiency anaemia & 462102 & 4719 & 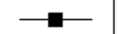 & & $0.85(0.77,0.94)$ & 0.003 \\
\hline Diabetes & 442740 & 9571 & & $\rightarrow-$ & $1.30(1.20,1.42)$ & $<0.001$ \\
\hline Carpal tunnel syndrome & 458489 & 5048 & & $=-$ & $1.05(0.95,1.17)$ & 0.349 \\
\hline Cataracts & 425971 & 16113 & $\rightarrow$ & & $0.93(0.87,0.99)$ & 0.015 \\
\hline \multirow[t]{2}{*}{ Cellulitis } & 463609 & 3409 & & $=$ & $1.02(0.89,1.17)$ & 0.784 \\
\hline & & 0. & 1 & 1.3 & & \\
\hline
\end{tabular}

Fig. 1 Risk of 25 common conditions per 70 grams/day (g/d) higher daily intake of unprocessed red and processed meat. Stratified for sex, age group, and region and adjusted for age (underlying time variable), race (4 groups where possible: White, Asian or Asian British, Black or Black British, mixed race or other, unknown), deprivation (Townsend index quintiles, unknown), qualification (college or university degree/vocational qualification, national examination at ages 17-18, national examination at age 16, others/unknown), employment (in paid employment, receiving pension, not in paid employment, unknown), smoking (never, former, current $<15$ cigarettes/day, current $>15$ cigarettes/day, current unknown amount of cigarettes/day, unknown), physical activity ( $<10$ excess METs per/week, 10 to $<50$ excess METs per/week, $\geq 50$ excess METs per/week, unknown), alcohol intake (none, $<1 \mathrm{~g} /$ day, 1 to $<10 \mathrm{~g} /$ day, 10 to $<20 \mathrm{~g} /$ day, $\geq 20 \mathrm{~g} /$ day, unknown), total fruit and vegetable intake ( $<3$ servings/ day, 3 to $<4$ servings/day, 4 to $<6$ servings/day, $\geq 6$ servings/day, unknown), cereal fibre score (sex-specific quintiles, unknown), oily fish intake ( 0 time/week, $<1$ time/week, 1 time/week, $\geq 2$ times/week, unknown), non-oily fish intake ( $<1$ time/week, 1 time/week, $\geq 2$ times/week, unknown), BMI (sex-specific quintiles, unknown), in women: menopausal status (pre-, postmenopausal, unknown), HRT (never, past, current, unknown), OCP use (never, past, current, unknown), and parity (nulliparous, 1-2, $\geq 3$, unknown). BMI body mass index, HRT hormone replacement therapy, OCP oral contraceptive pill, GERD Gastro-oesophageal reflux disease. $P$ trend in bold indicates $P$ value robust to Bonferroni correction $(P<0.002)$

consumption appear to account for a substantial part of the increased risks, suggesting that residual confounding by adiposity may still operate. We also observed inverse associations between higher intakes of unprocessed red meat and poultry meat and IDA, which were minimally affected by adjustment for BMI.

\section{Circulatory diseases}

Similar to our findings, a recent meta-analysis of prospective studies [6] and a recent prospective study from the Pan-European EPIC cohort which included over 7000 IHD cases [9] reported positive associations between unprocessed red meat and processed meat consumption and risk of IHD. For stroke, previous metaanalyses of prospective studies $[22,23]$ and a recent prospective study from the EPIC cohort [24] both reported null associations for unprocessed red and processed meat intake and haemorrhagic stroke; this is consistent with our main findings but not with our findings in participants diagnosed after 4 or more years of follow-up, 


\begin{tabular}{|c|c|c|c|c|c|}
\hline Disease subgroup & Total $\mathbf{N}$ & Cases & & HR $(95 \% \mathrm{Cl})$ per $50 \mathrm{~g} / \mathrm{d}$ & Ptrend \\
\hline \multicolumn{6}{|l|}{ Circulatory disease } \\
\hline Ischemic heart disease & 431229 & 13134 & $=-$ & $1.16(1.08,1.25)$ & $<0.001$ \\
\hline Atrial fibrillation and flutter & 431229 & 4745 & & $0.86(0.76,0.97)$ & 0.013 \\
\hline Cerebrovascular disease & 431229 & 5341 & $=$ & $1.06(0.94,1.18)$ & 0.348 \\
\hline Ischaemic stroke & 431229 & 2344 & & $1.05(0.89,1.25)$ & 0.555 \\
\hline Haemorrhagic stroke & 431229 & 941 & - & $1.08(0.83,1.41)$ & 0.554 \\
\hline Venous thromboembolism & 457969 & 3759 & $=-$ & $1.05(0.92,1.20)$ & 0.474 \\
\hline Varicose veins & 446070 & 2636 & & $0.95(0.81,1.10)$ & 0.467 \\
\hline Haemorrhoids & 448675 & 8305 & & $1.07(0.98,1.17)$ & 0.131 \\
\hline \multicolumn{6}{|l|}{ Respiratory disease } \\
\hline Pneumonia & 459886 & 6367 & $\longrightarrow-$ & $1.22(1.10,1.35)$ & $<0.001$ \\
\hline \multicolumn{6}{|l|}{ Digestive disease } \\
\hline GERD & 440391 & 7023 & & $0.99(0.90,1.08)$ & 0.758 \\
\hline Gastritis and duodenitis & 451544 & 11380 & & $0.96(0.89,1.03)$ & 0.296 \\
\hline Inguinal hernia & 441323 & 8028 & - & $1.00(0.91,1.10)$ & 0.987 \\
\hline Noninfective enteritis and colitis & 454181 & 6241 & - & $1.03(0.93,1.14)$ & 0.572 \\
\hline Diverticular disease & 455843 & 12829 & $\rightarrow-$ & $1.17(1.09,1.26)$ & $<0.001$ \\
\hline Colon polyps & 446601 & 37430 & & $1.08(1.04,1.13)$ & $<0.001$ \\
\hline Gallbladder disease & 448946 & 8930 & & $1.04(0.96,1.14)$ & 0.320 \\
\hline \multicolumn{6}{|l|}{ Joint disorder } \\
\hline Osteoarthritis & 417663 & 18095 & & $0.96(0.90,1.02)$ & 0.182 \\
\hline \multicolumn{6}{|l|}{ Genitourinary disease } \\
\hline Kidney stones & 461138 & 2450 & 1 & $1.00(0.85,1.17)$ & 0.956 \\
\hline Urinary tract infection & 460551 & 5216 & $\square$ & $1.09(0.98,1.22)$ & 0.114 \\
\hline Enlarged prostate & 205842 & 3548 & $=$ & $1.01(0.88,1.17)$ & 0.849 \\
\hline Female genital prolapse & 239957 & 6069 & - & $1.08(0.97,1.19)$ & 0.157 \\
\hline \multicolumn{6}{|l|}{ Other diseases } \\
\hline Uterine fibroids & 182003 & 9266 & 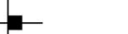 & $1.02(0.95,1.10)$ & 0.592 \\
\hline Iron deficiency anaemia & 462674 & $\longrightarrow-$ & & $0.80(0.72,0.90)$ & $<0.001$ \\
\hline Diabetes & 443255 & 9595 & $\rightarrow$ & $1.21(1.11,1.32)$ & $<0.001$ \\
\hline Carpal tunnel syndrome & 459061 & 5055 & $=-$ & $1.05(0.94,1.18)$ & 0.357 \\
\hline Cataracts & 426463 & 16141 & & $0.93(0.88,1.00)$ & 0.037 \\
\hline \multirow[t]{3}{*}{ Cellulitis } & 464191 & 3414 & & $0.93(0.81,1.07)$ & 0.301 \\
\hline & & 0.7 & 1.3 & & \\
\hline & & Unpro & & & \\
\hline
\end{tabular}

Fig. 2 Risk of 25 common conditions per $50 \mathrm{grams} /$ day (g/d) higher daily intake of unprocessed red meat. Stratified for sex, age group, and region and adjusted for age (underlying time variable), race (4 groups where possible: White, Asian or Asian British, Black or Black British, mixed race or others, unknown), deprivation (Townsend index quintiles, unknown), qualification (college or university degree/vocational qualification, national examination at ages 17-18, national examination at age 16, others/unknown), employment (in paid employment, receiving pension, not in paid employment, unknown), smoking (never, former, current $<15$ cigarettes/day, current $>15$ cigarettes/day, current unknown amount of cigarettes/day, unknown), physical activity ( $<10$ excess METs per/week, 10 to $<50$ excess METs per/week, $\geq 50$ excess METs per/week, unknown), alcohol intake (none, $<1 \mathrm{~g} /$ day, 1 to $<10 \mathrm{~g} /$ day, 10 to $<20 \mathrm{~g} /$ day, $\geq 20 \mathrm{~g} /$ day, unknown), total fruit and vegetable intake $(<3$ servings/day, 3 to $<$ 4 servings/day, 4 to $<6$ servings/day, $\geq 6$ servings/day, unknown), cereal fibre score (sex-specific quintiles, unknown), oily fish intake ( 0 time/ week, $<1$ time/week, 1 time/week, $\geq 2$ times/week, unknown), non-oily fish intake ( $<1$ time/week, 1 time/week, $\geq 2$ times/week, unknown), BMl (sex-specific quintiles, unknown), in women: menopausal status (pre-, postmenopausal, unknown), HRT (never, passt, current, unknown), OCP use (never, past, current, unknown), and parity (nulliparous, $1-2, \geq 3$, unknown). BMI body mass index, HRT hormone replacement therapy, OCP oral contraceptive pill, GERD Gastro-oesophageal reflux disease. $P$ trend in bold indicates $P$ value robust to Bonferroni correction $(P<0.002)$

although this might be a chance finding due to shorter follow-up. Processed meats contain high amounts of sodium [25], a risk factor for high blood pressure [26], which is a causal risk factor for IHD and stroke [27]. Furthermore, unprocessed red meat and processed meat are major dietary sources of saturated fatty acids (SFAs) which can increase low-density lipoprotein (LDL) cholesterol, an established causal risk factor for IHD [28]. It is also possible that the positive association we observed for unprocessed red meat intake and IHD risk might relate to gut microbiota metabolism, for example through the production of trimethylamine- $N$-oxide [25-27], but the importance of this potential pathway is uncertain.

\section{Respiratory disease}

Higher consumption of unprocessed red and processed meat was associated with a higher risk of pneumonia. To the best of our knowledge, these associations have not been shown previously, except for one recent study that found that higher intake of red meat (both processed and unprocessed) was associated with a higher risk of death due to respiratory disease, which included 


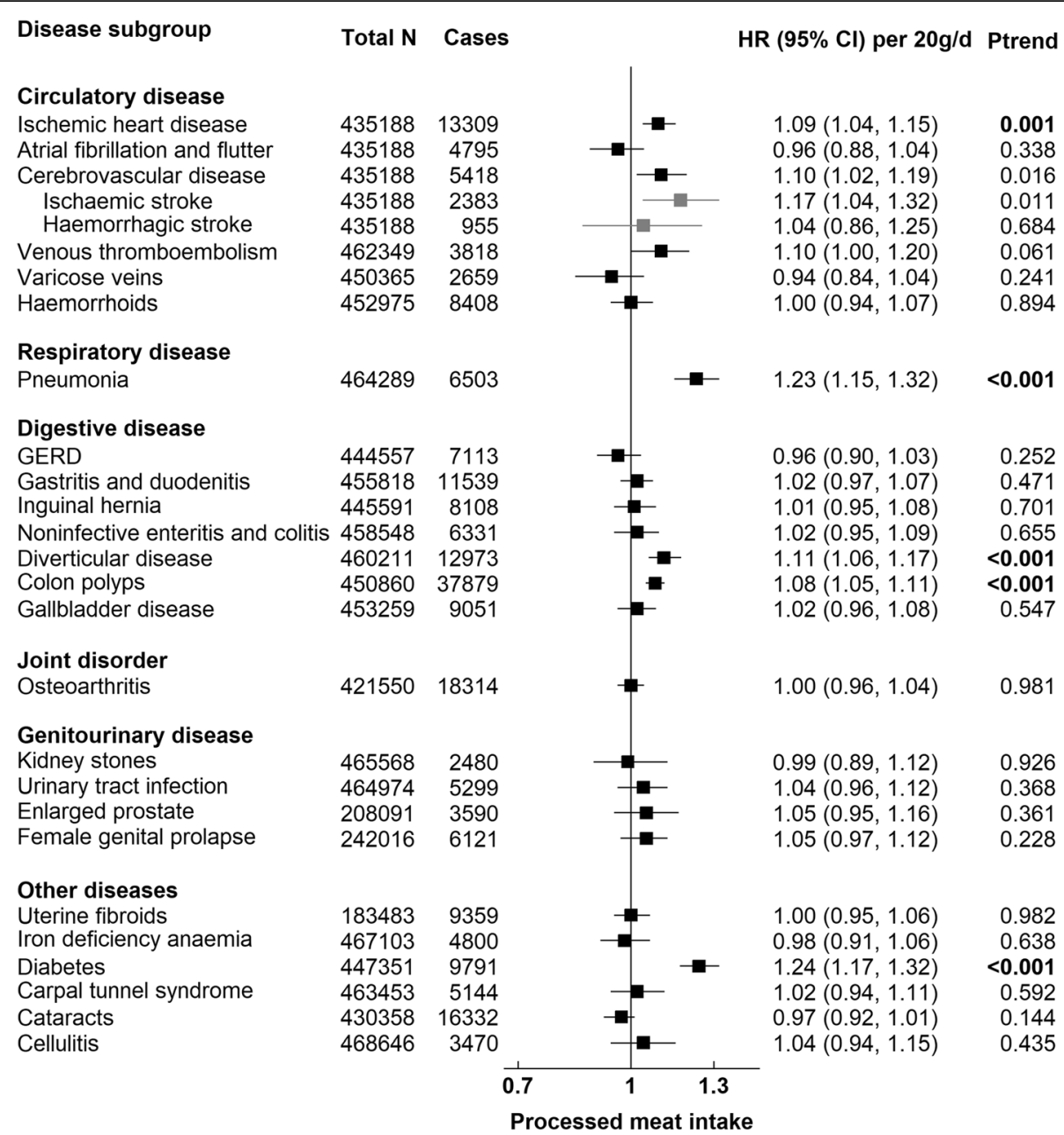

Fig. 3 Risk of 25 common conditions per $20 \mathrm{grams} /$ day (g/d) higher daily intake of processed meat. Stratified for sex, age group, and region and adjusted for age (underlying time variable), race (4 groups where possible: White, Asian or Asian British, Black or Black British, mixed race or others, unknown), deprivation (Townsend index quintiles, unknown), qualification (college or university degree/vocational qualification, national examination at ages 17-18, national examination at age 16, others/unknown), employment (in paid employment, receiving pension, not in paid employment, unknown), smoking (never, former, current $<15$ cigarettes/day, current $\geq 15$ cigarettes/day, current unknown amount of cigarettes/ day, unknown), physical activity (< 10 excess METs per/week, 10 to $<50$ excess METs per/week, $\geq 50$ excess METs per/week, unknown), alcohol intake (none, $<1 \mathrm{~g} /$ day, 1 to $<10 \mathrm{~g} /$ day, 10 to $<20 \mathrm{~g} /$ day, $\geq 20 \mathrm{~g} /$ day, unknown), total fruit and vegetable intake $(<3$ servings/day, 3 to $<4$ servings/day, 4 to $<6$ servings/day, $\geq 6$ servings/day, unknown), cereal fibre score (sex-specific quintiles, unknown), oily fish intake (0 time/week, $<1$ time/week, 1 time/week, $>2$ times/week, unknown), non-oily fish intake ( $<1$ time/week, 1 time/week, $>2$ times/week, unknown), BMI (sexspecific quintiles, unknown), in women: menopausal status (pre-, postmenopausal, unknown), HRT (never, past, current, unknown), OCP use (never, past, current, unknown), and parity (nulliparous, 1-2, $\geq 3$, unknown). BMI body mass index, HRT hormone replacement therapy, OCP oral contraceptive pill, GERD Gastro-oesophageal reflux disease. $P$ trend in bold indicates $P$ value robust to Bonferroni correction $(P<0.002)$

pneumonia [21]. It is possible that the observed association might reflect a causal link, for example related to the high availability of iron in unprocessed red and processed meat (see further discussion below in relation to anaemia), since excess iron has been found to be associated with a higher risk of infection [29] and increased availability of iron for pathogens [30]. It is also possible that hospital admission for pneumonia is a marker for co-morbidity and overall frailty [31]; therefore, residual confounding might operate (see further discussion on residual confounding below).

\section{Digestive diseases}

Few prospective studies have examined the risk for diverticular disease $[32,33]$, but consistent with our findings, the Health Professionals Follow-up Study (HPFS) observed increased risks of incident diverticulitis with higher consumption of unprocessed red and processed meat [32]. The HPFS did not observe an association for poultry meat, but had lower power than the current study. Meat consumption might affect the risk of diverticular disease via the intestinal microbiome, by altering microbial community structure and metabolism [34]. 


\begin{tabular}{|c|c|c|c|c|c|}
\hline Disease subgroup & Total $\mathbf{N}$ & Cases & & HR $(95 \% \mathrm{Cl})$ per $30 \mathrm{~g} / \mathrm{d}$ & Ptrend \\
\hline \multicolumn{6}{|l|}{ Circulatory disease } \\
\hline $\begin{array}{l}\text { Iscnemic neart disease } \\
\text { Atrial fibrillation and flutter }\end{array}$ & $\begin{array}{l}435331 \\
435337\end{array}$ & $\begin{array}{r}13299 \\
4793\end{array}$ & $\rightarrow-$ & $\begin{array}{l}1.08(1.02,1.14) \\
1.08(0.99,1.18)\end{array}$ & $\begin{array}{l}0.008 \\
0.101\end{array}$ \\
\hline Cerebrovascular disease & 435337 & 5422 & - & $1.06(0.97,1.15)$ & 0.200 \\
\hline Ischaemic stroke & 435337 & 2388 & - & $1.03(0.91,1.17)$ & 0.644 \\
\hline Haemorrhagic stroke & 435337 & 957 & $=$ & $1.07(0.88,1.31)$ & 0.499 \\
\hline Venous thromboembolism & 462512 & 3818 & $\rightarrow$ & $1.02(0.92,1.13)$ & 0.686 \\
\hline Varicose veins & 450538 & 2661 & 5 & $1.00(0.88,1.13)$ & 0.944 \\
\hline Haemorrhoids & 453135 & 8415 & & $1.07(1.00,1.15)$ & 0.054 \\
\hline \multicolumn{6}{|l|}{ Respiratory disease } \\
\hline Pneumonia & 464457 & 6505 & - & $1.03(0.96,1.11)$ & 0.424 \\
\hline \multicolumn{6}{|l|}{ Digestive disease } \\
\hline GERD & 444701 & 7121 & $\rightarrow-$ & $1.17(1.09,1.26)$ & $<0.001$ \\
\hline Gastritis and duodenitis & 455977 & 11535 & - & $1.12(1.05,1.18)$ & $<0.001$ \\
\hline Inguinal hernia & 445770 & 8110 & & $1.01(0.94,1.08)$ & 0.868 \\
\hline Noninfective enteritis and colitis & 458709 & 6332 & - & $1.04(0.96,1.13)$ & 0.311 \\
\hline Diverticular disease & 460367 & 12983 & $\rightarrow-$ & $1.10(1.04,1.17)$ & 0.001 \\
\hline Colon polyps & 451024 & 37860 & $=$ & $1.05(1.01,1.08)$ & 0.005 \\
\hline Gallbladder disease & 453426 & 9055 & $\rightarrow-$ & $1.11(1.04,1.19)$ & $<0.001$ \\
\hline \multicolumn{6}{|l|}{ Joint disorder } \\
\hline Osteoarthritis & 421695 & 18304 & $=$ & $1.07(1.03,1.13)$ & 0.003 \\
\hline \multicolumn{6}{|l|}{ Genitourinary disease } \\
\hline Kidney stones & 465737 & 2483 & $\because$ & $1.13(0.99,1.28)$ & 0.062 \\
\hline Urinary tract infection & 465129 & 5302 & - & $0.97(0.89,1.05)$ & 0.445 \\
\hline Enlarged prostate & 208113 & 3592 & $=$ & $1.03(0.92,1.14)$ & 0.642 \\
\hline Female genital prolapse & 242161 & 6126 & - & $1.08(0.99,1.17)$ & 0.071 \\
\hline \multicolumn{6}{|l|}{ Other diseases } \\
\hline Uterine fibroids & 183580 & 9365 & & $1.01(0.95,1.08)$ & 0.666 \\
\hline Iron deficiency anaemia & 467264 & 4796 & & $0.83(0.76,0.90)$ & $<0.001$ \\
\hline Diabetes & 447503 & 9799 & $\rightarrow$ & $1.14(1.07,1.21)$ & $<0.001$ \\
\hline Carpal tunnel syndrome & 463611 & 5146 & - & $1.04(0.95,1.13)$ & 0.419 \\
\hline Cataracts & 430519 & 16349 & - & $0.96(0.92,1.01)$ & 0.119 \\
\hline \multirow[t]{2}{*}{ Cellulitis } & 468817 & 3464 & - & $1.03(0.92,1.14)$ & 0.616 \\
\hline & & 0.7 & 1.3 & & \\
\hline \multicolumn{6}{|c|}{ Poultry meat intake } \\
\hline
\end{tabular}

Fig. 4 Risk of 25 common conditions per 30 grams/day (g/d) higher daily intake of poultry meat. Stratified for sex, age group, and region and adjusted for age (underlying time variable), race (4 groups where possible: White, Asian or Asian British, Black or Black British, mixed race or others, unknown), deprivation (Townsend index quintiles, unknown), qualification (college or university degree/vocational qualification, national examination at ages 17-18, national examination at age 16, other/unknown), employment (in paid employment, receiving pension, not in paid employment, unknown), smoking (never, former, current $<15$ cigarettes/day, current $>15$ cigarettes/day, current unknown amount of cigarettes/ day, unknown), physical activity (< 10 excess METs per/week, 10 to $<50$ excess METs per/week, $\geq 50$ excess METs per/week, unknown), alcohol intake (none, $<1 \mathrm{~g} /$ day, 1 to $<10 \mathrm{~g} /$ day, 10 to $<20 \mathrm{~g} /$ day, $\geq 20 \mathrm{~g} /$ day, unknown), total fruit and vegetable intake $(<3$ servings/day, 3 to $<4$ servings/day, 4 to $<6$ servings/day, $\geq 6$ servings/day, unknown), cereal fibre score (sex-specific quintiles, unknown), oily fish intake (0 time/week, $<1$ time/week, 1 time/week, $\geq 2$ times/week, unknown), non-oily fish intake ( $<1$ time/week, 1 time/week, $\geq 2$ times/week, unknown), BMI (sexspecific quintiles, unknown), in women: menopausal status (pre-, postmenopausal, unknown), HRT (never, past, current, unknown), OCP use (never, past, current, unknown), and parity (nulliparous, $1-2, \geq 3$, unknown). BMI body mass index, HRT hormone replacement therapy, OCP oral contraceptive pill, GERD Gastro-oesophageal reflux disease. $P$ trend in bold indicates $P$ value robust to Bonferroni correction $(P<0.002)$

A recent meta-analysis of prospective studies reported that unprocessed red and processed meat consumption was positively associated with the risk of colorectal adenomas [35], which is consistent with our findings for colon polyps. Unprocessed red meat is a source of heme iron and processed meat usually contains nitrite and nitrates; these can increase the formation of $\mathrm{N}$-nitroso compounds [36], which are mutagenic and have been associated with a higher risk of colorectal adenomas [37].

To our knowledge, this is the first prospective study of meat consumption and risk of GERD and gastritis and duodenitis. We found a positive association between poultry meat intake and GERD risk, whereas the available cross-sectional evidence suggests a null association for meat (total) [38-41]. We also found a positive association between poultry meat consumption and risk of gastritis and duodenitis. Helicobacter pylori, a bacterium that increases the risk of gastritis [42], has been previously detected in raw poultry meat [43]. Therefore, it is possible that the observed association might relate to inappropriate handling or cooking of poultry meat, but additional research is needed. 
Some published studies have found evidence of an association between higher unprocessed red and processed meat consumption and gallbladder disease which remained after BMI adjustment $[16,17]$, whereas in our analyses this association was greatly attenuated and not significant after adjusting for BMI. In the present study, BMI was calculated from standardised measurements of weight and height, whereas previous studies used selfreported weight and height. Therefore, it is possible that adjusting for BMI in this study explained a larger proportion of the observed associations; high BMI has been consistently shown to be associated with a large increase in the risk of gallbladder disease in both observational and genetic studies [18-20]. We observed a novel association between poultry intake and gallbladder disease, though additional research is needed to assess this association.

\section{Other diseases}

We found an inverse association between the consumption of unprocessed red meat and poultry meat and risk of IDA. Some previous evidence from prospective studies [44] supports these findings and has also shown a positive association between unprocessed red meat [45] and total meat [46-48] consumption and indicators of body iron stores. Moreover, previous cross-sectional work from the UK Biobank has shown that people who did not consume meat were more likely to be anaemic [49]. This association is likely related to the high availability of heme iron in meat, which is more easily absorbed than non-heme iron [50].

Similar to our findings, meta-analyses of prospective cohort studies have consistently reported a positive association between unprocessed red and processed meat consumption and risk of diabetes [7, 51, 52]. We also found a positive association between poultry meat consumption and risk of diabetes, which has been reported in some [14] but not all prospective studies [13, 53]. Obesity is the major risk factor for diabetes, and the association for unprocessed and processed meat intake (combined) and diabetes in the present study was substantially attenuated (by $\sim 60 \%$ ) after adjusting for BMI, suggesting that the remaining association with meat may be entirely due to higher adiposity. It is also possible that meat could affect risk independently of adiposity; for example, high intakes of heme iron and greater iron storage may promote the formation of hydroxyl radicals that damage the pancreatic beta cells, thereby impairing insulin synthesis and excretion [54, 55].

\section{Role of BMI}

In the present study, most of the positive associations between meat consumption and health risks were substantially attenuated after adjusting for BMI, suggesting that BMI was a strong confounder or possible mediator for many of the meat and disease associations. BMI is an important risk factor for many of the diseases examined (e.g. diabetes [7]). BMI was highest in participants who consumed meat most frequently, and some previous studies have found that high meat consumption is associated with weight gain $[56,57]$, but it is unclear whether this indicates any specific impact of meat or an association in these populations of high meat intakes with high total energy intakes. The associations of meat with disease risk reported here which remain after adjustment for BMI might still be due to higher adiposity, because BMI is not a perfect measure of this characteristic; we observed similar effects when adjusting for waist circumference (results not shown), but, as with BMI, waist circumference is not a perfect measure of adiposity and there could still be residual confounding.

\section{Strengths and limitations}

As far as we are aware, this is the first outcome-wide study of meat intake and risk of 25 common conditions (other than cancer). Additional strengths of this study include the large size of the cohort, its prospective design, and the comprehensive array of confounders considered. This allowed us to investigate a large number of common conditions and thus avoid outcome selection bias, while simultaneously controlling for confounding. Additionally, we used national record linkage to ascertain information on disease incidence, which is objective and minimises selective loss to follow-up. Nevertheless, some potential methodological issues should be considered when interpreting our findings. Some measurement error would have occurred while measuring meat consumption at baseline; however, we reduced the impact of random error and short-term variation in diet by using the repeated 24-h recall WebQ data and applying corrected intakes to each category of the baseline intakes. Another limitation was that the touchscreen dietary questionnaire only included a subset of food groups and food items and therefore total dietary intake could not be calculated, and confounding by energy balance could not be directly accounted for. We addressed this by adjusting for BMI, physical activity, and other dietary factors [24]; however, there might still be some residual confounding by energy intake. Likewise, participants who consumed high amounts of unprocessed red meat also consumed high amounts of processed meat. Therefore, we could not mutually adjust the meat types, and there may be residual confounding. Multiple testing might have led to some spurious findings; we addressed this by using Bonferroni correction, but this is a stringent approach and it is possible that some real associations did not meet the Bonferroni threshold. Another consideration is the use of hospital records for incident case ascertainment. Some 
conditions might only require hospital use at later stages (e.g. diabetes), and therefore, some admissions might reflect prevalent and/or more severe cases. Finally, given the observational nature of this study, it is possible that there is still unmeasured confounding, residual confounding, and reverse causality. For instance, in analyses restricted to never smokers, some of the adjusted risk estimates were lower than in the main analysis (e.g. for unprocessed red meat intake and diabetes and for processed meat intake and IHD), suggesting that even after adjustment for smoking there may be residual confounding. However, most of our results were similar after excluding participants who smoked or formerly smoked and after excluding the first 4 years of follow-up.

\section{Conclusions}

Our findings from this large, prospective study of British adults show that meat consumption is associated with higher risks of several common conditions but a lower risk of IDA. The higher risks are at least partly accounted for by higher BMI, and some of the associations remaining after adjusting for BMI or waist circumference may still be due to other aspects of adiposity. Additional research is needed to evaluate whether these differences in risk reflect causal relationships, and if so what proportion of incident cases for these different outcomes that could be prevented by decreasing meat consumption.

\section{Supplementary Information}

The online version contains supplementary material available at https://doi. org/10.1186/s12916-021-01922-9.

Additional file $\mathbf{1}$ Methods 1. Assessment of dietary intake for meat. Methods 2. Assessment of health outcomes. Methods 3. Covariates. Table 1. Disease outcome definition and exclusion criteria. Table 2. Baseline characteristics of participants by total meat intake in UK Biobank ( $n=467,384)$. Table 3. Baseline characteristics of participants by unprocessed red meat intake in UK Biobank $(n=468,328)$. Table 4. Baseline characteristics of participants by processed meat intake in UK Biobank ( $n=472,844)$. Table 5. Baseline characteristics of participants by poultry meat intake in UK Biobank $(n=473,011)$. Table 6 . Risk of 25 common conditions by total meat intake in UK Biobank. Table 7. Risk of 25 common conditions by unprocessed red and processed meat intake in UK Biobank. Table 8. Risk of 25 common conditions by unprocessed red meat intake in UK Biobank. Table 9. Risk of 25 common conditions by processed meat intake in UK Biobank. Table 10. Risk of 25 common conditions by poultry meat intake in UK Biobank. Figure 1. Participant flow chart of the study. Figure 2. Risk of 25 common conditions by higher daily intake of total meat excluding the first 4 years of follow-up, in never smokers. Figure 3. Risk of 25 common conditions by higher daily intake of unprocessed red and processed meat excluding the first 4 years of follow-up, in never smokers. Figure 4. Risk of 25 common conditions by higher daily intake of unprocessed red meat excluding the first 4 years of follow-up, in never smokers. Figure 5. Risk of 25 common conditions by higher daily intake of processed meat excluding the first 4 years of follow-up, in never smokers. Figure 6. Risk of 25 common conditions by higher daily intake of poultry meat excluding the first 4 years of follow-up, in never smokers. Figure 7. Risk of 25 common conditions per $100 \mathrm{~g} /$ day higher daily intake of total meat.

\section{Abbreviations}

BMl: Body mass index; Cl: Confidence interval; GERD: Gastro-oesophageal reflux disease; HR: Hazard ratio; HRT: Hormone replacement therapy; ICD: International Statistical Classification of Diseases; IDA: Iron deficiency anaemia; IHD: Ischaemic heart disease; MET: Metabolic equivalent; OCP: Oral contraceptive pill

\section{Acknowledgements}

This research was conducted using the UK Biobank Resource under application number 24494. We thank all participants, researchers, and support staff who make the study possible.

\section{Authors' contributions}

KP, TJK, and AP-C conceived and designed the research question; AP-C managed the project and was responsible for acquiring the data; KP analysed the data, prepared the figures and tables, and wrote the first draft of the manuscript; GKF, AK, and KP prepared the data for analysis; and GKF, AK, PNA, TYNT, JAS, RCT, TJK, and AP-C provided input on data analysis and interpretation of results. All authors revised the manuscript critically for important intellectual content and read and approved the final manuscript.

\section{Funding}

This work was supported by the Wellcome Trust, Our Planet Our Health (Livestock, Environment and People - LEAP) [grant number 205212/Z/16/Z]; Cancer Research UK [grant numbers C8211/A19170 and C8211/A29017]; and the UK Medical Research Council [grant number MR/M012190/1]. AP-C is supported by a Cancer Research UK Population Research Fellowship [grant number C60192/A28516] and by the World Cancer Research Fund (WCRF UK), as part of the WCRF International grant programme [grant number 2019/1953].

\section{Availability of data and materials}

The datasets generated/and or analysed in the current study will be made available for bona fide researchers who apply to use the UK Biobank data set by registering and applying at http://www.ukbiobank.ac.uk/register-apply.

\section{Ethics approval and consent to participate}

The study was approved by The National Information Governance Board for Health and Social Care and the National Health Service North West Multicentre Research Ethics Committee (reference number 06/MRE08/65), and participants provided informed consent at baseline and to be followed up using data linkage. The UK Biobank protocol is available online (http:// www.ukbiobank.ac.uk/wp-content/uploads/2011/11/UK-Biobank-Protocol. pdf).

\section{Consent for publication}

Not applicable

\section{Competing interests}

The authors declare no competing interests.

\section{Author details}

${ }^{1}$ Cancer Epidemiology Unit, Nuffield Department of Population Health, University of Oxford, Richard Doll Building, Old Road Campus, Oxford OX3 7LF, UK. ${ }^{2}$ Department of International Development, University of Oxford, 3 Mansfield Rd, Oxford OX1 3TB, UK.

Received: 26 August 2020 Accepted: 20 January 2021

Published online: 02 March 2021

\section{References}

1. Bouvard V, Loomis D, Guyton KZ, Grosse Y, Ghissassi FE, Benbrahim-Tallaa L, et al. Carcinogenicity of consumption of red and processed meat. Lancet Oncol. 2015;16(16):1599-600. https://doi.org/10.1016/s1470-2045(15)00444-1.

2. Public Health England. The Eatwell guide. London: Public Health England; 2016.

3. World Cancer Research Fund; American Institute for Cancer Research. World Cancer Research Fund; American Institute for Cancer Research. Meat, fish and dairy products and the risk of cancer. Continuous Update Project Expert Report. 2018. 
4. Knuppel A, Papier K, Fensom GK, Appleby PN, Schmidt JA, Tong TYN, et al. Meat intake and cancer risk: prospective analyses in UK Biobank. Int J Epidemiol. 2020. https://doi.org/10.1093/ije/dyaa142.

5. Etemadi A, Sinha R, Ward MH, Graubard BI, Inoue-Choi M, Dawsey SM, et al. Mortality from different causes associated with meat, heme iron, nitrates, and nitrites in the NIH-AARP Diet and Health Study: population based cohort study. BMJ (Clinical research ed). 2017;357(j1957). https://doi.org/10. 1136/bmj.j1957.

6. Bechthold A, Boeing H, Schwedhelm C, Hoffmann G, Knuppel S, Iqbal K, et al. Food groups and risk of coronary heart disease, stroke and heart failure: a systematic review and dose-response meta-analysis of prospective studies. Crit Rev Food Sci Nutr. 2019;59(7):1071-90. https://doi.org/10.1080/ 10408398.2017.1392288.

7. Pan A, Sun Q, Bernstein AM, Schulze MB, Manson JE, Willett WC, et al. Red meat consumption and risk of type 2 diabetes: 3 cohorts of US adults and an updated meta-analysis. Am J Clin Nutr. 2011;94(4):1088-96. https://doi. org/10.3945/ajcn.111.018978.

8. Abete I, Romaguera D, Vieira AR, Lopez de Munain A, Norat T. Association between total, processed, red and white meat consumption and all-cause, CVD and IHD mortality: a meta-analysis of cohort studies. Br J Nutr. 2014; 112(5):762-75. https://doi.org/10.1017/s000711451400124x.

9. Key TJ, Appleby PN, Bradbury KE, Sweeting M, Wood A, Johansson I, et al. Consumption of meat, fish, dairy products, and eggs and risk of ischemic heart disease. Circulation. 2019;139(25):2835-45. https://doi.org/10.1161/ circulationaha.118.038813.

10. Micha R, Wallace SK, Mozaffarian D. Red and processed meat consumption and risk of incident coronary heart disease, stroke, and diabetes mellitus: a systematic review and meta-analysis. Circulation. 2010;121(21):2271-83. https://doi.org/10.1161/circulationaha.109.924977.

11. lacoviello L, Bonaccio M, Cairella G, Catani MV, Costanzo S, D'Elia L, et al. Diet and primary prevention of stroke: systematic review and dietary recommendations by the ad hoc Working Group of the Italian Society of Human Nutrition. Nutrition, metabolism, Cardiovascular Dis. 2018;28(4):30934. https://doi.org/10.1016/j.numecd.2017.12.010

12. Mohammadi H, Jayedi A, Ghaedi E, Golbidi D, Shab-Bidar S. Dietary poultry intake and the risk of stroke: a dose-response meta-analysis of prospective cohort studies. Clin Nutrition ESPEN. 2018;23:25-33. https://doi.org/10.1016/j. clnesp.2017.11.001.

13. Feskens EJ, Sluik D, van Woudenbergh GJ. Meat consumption, diabetes, and its complications. Current diabetes reports. 2013;13(2):298-306. https://doi. org/10.1007/s11892-013-0365-0.

14. Talaei M, Wang YL, Yuan JM, Pan A, Koh WP. Meat, dietary heme iron, and risk of type 2 diabetes mellitus: the Singapore Chinese Health Study. Am J Epidemiol. 2017;186(7):824-33. https://doi.org/10.1093/aje/kwx156.

15. Williamson PR, Gamble C, Altman DG, Hutton JL. Outcome selection bias in meta-analysis. Stat Methods Med Res. 2005;14(5):515-24. https://doi.org/10. 1191/0962280205sm415oa

16. VanderWeele TJ. Outcome-wide epidemiology. Epidemiology (Cambridge, Mass). 2017;28(3):399-402. https://doi.org/10.1097/ede.0000000000000641.

17. Fry A, Littlejohns TJ, Sudlow C, Doherty N, Adamska L, Sprosen T, et al. Comparison of sociodemographic and health-related characteristics of UK Biobank participants with those of the general population. Am J Epidemiol. 2017;186(9):1026-34. https://doi.org/10.1093/aje/kw×246.

18. Greenwood DC, Hardie L, Frost GS, Alwan NA, Bradbury KE, Carter M, et al. Validation of the Oxford WebQ online 24-hour dietary questionnaire using biomarkers. Am J Epidemiol. 2019;188(10):1858-67. https://doi.org/10.1093/ aje/kwz165.

19. Key TJ, Balkwill A, Bradbury KE, Reeves GK, Kuan AS, Simpson RF, et al. Foods, macronutrients and breast cancer risk in postmenopausal women: a large UK cohort. Int J Epidemiol. 2019;48(2):489-500. https://doi.org/10.1093/ije/dyy238.

20. Townsend P, Phillimore P, Beattie A. Health and deprivation: inequality and the North. London: Croom Helm; 1988.

21. Bradbury KE, Young HJ, Guo W, Key TJ. Dietary assessment in UK Biobank: an evaluation of the performance of the touchscreen dietary questionnaire. J Nutritional Sci. 2018;7:e6. https://doi.org/10.1017/jns.2017.66.

22. Chen GC, Lv DB, Pang Z, Liu QF. Red and processed meat consumption and risk of stroke: a meta-analysis of prospective cohort studies. Eur J Clin Nutr. 2013;67(1):91-5. https://doi.org/10.1038/ejcn.2012.180.

23. Kaluza J, Wolk A, Larsson SC. Red meat consumption and risk of stroke: a meta-analysis of prospective studies. Stroke. 2012;43(10):2556-60. https://doi. org/10.1161/strokeaha.112.663286.
24. Tong TYN, Appleby PN, Key TJ, Dahm CC, Overvad K, Olsen A, et al. The associations of major foods and fibre with risks of ischaemic and haemorrhagic stroke: a prospective study of 418329 participants in the EPIC cohort across nine European countries. Eur Heart J. 2020;41(28):2632-40. https://doi.org/10.1093/eurheartj/ehaa007.

25. Micha R, Michas G, Mozaffarian D. Unprocessed red and processed meats and risk of coronary artery disease and type 2 diabetes--an updated review of the evidence. Curr Atheroscler Rep. 2012;14(6):515-24. https://doi.org/10. 1007/s11883-012-0282-8.

26. He FJ, MacGregor GA. Effect of modest salt reduction on blood pressure: a meta-analysis of randomized trials. Implications for public health. J Hum Hypertens. 2002;16(11):761-70. https://doi.org/10.1038/sj.jhh.1001459.

27. Rosendorff C, Lackland DT, Allison M, Aronow WS, Black HR, Blumenthal $\mathrm{RS}$, et al. Treatment of hypertension in patients with coronary artery disease: a scientific statement from the American Heart Association, American College of Cardiology, and American Society of Hypertension. J Am Soc Hypertension. 2015;9(6):453-98. https://doi.org/10.1016/j.jash. 2015.03.002.

28. Mensink Ronald. Effects of saturated fatty acids on serum lipids and lipoproteins: a systematic review and regression analysis,: World Health Organisation, 2016. https://www.who.int/nutrition/publications/ nutrientrequirements/sfa_systematic_review/en/.

29. Ganz T, Nemeth E. Iron homeostasis in host defence and inflammation. Nat Rev Immunol. 2015;15(8):500-10. https://doi.org/10.1038/nri3863.

30. Cassat JE, Skaar EP. Iron in infection and immunity. Cell Host Microbe. 2013; 13(5):509-19. https://doi.org/10.1016/j.chom.2013.04.010.

31. McDonald HI, Nitsch D, Millett ER, Sinclair A, Thomas SL. New estimates of the burden of acute community-acquired infections among older people with diabetes mellitus: a retrospective cohort study using linked electronic health records. Diabetic Med. 2014;31(5):606-14. https://doi.org/10.1111/ dme.12384.

32. Cao Y, Strate LL, Keeley BR, Tam I, Wu K, Giovannucci EL, et al. Meat intake and risk of diverticulitis among men. Gut. 2018;67(3):466-72. https://doi.org/ 10.1136/gutjnl-2016-313082

33. Crowe FL, Appleby PN, Allen NE, Key TJ. Diet and risk of diverticular disease in Oxford cohort of European Prospective Investigation into Cancer and Nutrition (EPIC): prospective study of British vegetarians and nonvegetarians. BMJ (Clinical research ed). 2011;343:d4131. https://doi.org/10. 1136/bmj.d4131.

34. David LA, Maurice CF, Carmody RN, Gootenberg DB, Button JE, Wolfe BE, et al. Diet rapidly and reproducibly alters the human gut microbiome. Nature. 2014;505(7484):559-63. https://doi.org/10.1038/nature12820.

35. Aune D, Chan DS, Vieira AR, Navarro Rosenblatt DA, Vieira R, Greenwood DC, et al. Red and processed meat intake and risk of colorectal adenomas: a systematic review and meta-analysis of epidemiological studies. Cancer Causes Control. 2013;24(4):611-27. https://doi.org/10.1007/s10552-012-0139-z.

36. Gamage SMK, Dissabandara L, Lam AK, Gopalan V. The role of heme iron molecules derived from red and processed meat in the pathogenesis of colorectal carcinoma. Crit Rev Oncol Hematol. 2018;126:121-8. https://doi. org/10.1016/j.critrevonc.2018.03.025.

37. Ward MH, Cross AJ, Divan H, Kulldorff M, Nowell-Kadlubar S, Kadlubar FF, et al. Processed meat intake, CYP2A6 activity and risk of colorectal adenoma. Carcinogenesis. 2007;28(6):1210-6. https://doi.org/10.1093/carcin/ bgm009.

38. Cela L, Kraja B, Hoti K, Toci E, Muja H, Roshi E, et al. Lifestyle characteristics and gastroesophageal reflux disease: a population-based study in Albania. Gastroenterol Res Pract. 2013;2013:936792. https://doi.org/10.1155/2013/ 936792.

39. Nam SY, Park BJ, Cho YA, Ryu KH, Choi IJ, Park S, et al. Different effects of dietary factors on reflux esophagitis and non-erosive reflux disease in 11,690 Korean subjects. J Gastroenterol. 2017;52(7):818-29. https://doi.org/10.1007/ s00535-016-1282-1.

40. Zheng Z, Nordenstedt H, Pedersen NL, Lagergren J, Ye W. Lifestyle factors and risk for symptomatic gastroesophageal reflux in monozygotic twins. Gastroenterology. 2007;132(1):87-95. https://doi.org/ 10.1053/j.gastro.2006.11.019

41. El-Serag HB, Satia JA, Rabeneck L. Dietary intake and the risk of gastrooesophageal reflux disease: a cross sectional study in volunteers. Gut. 2005; 54(1):11-7. https://doi.org/10.1136/gut.2004.040337.

42. Van Hecke T, Van Camp J, De Smet S. Oxidation during digestion of meat: interactions with the diet and helicobacter pylori gastritis, and implications 
on human health. Compr Rev Food Sci Food Saf. 2017;16(2):214-33. https:// doi.org/10.1111/1541-4337.12248.

43. Quaglia NC, Dambrosio A. Helicobacter pylori: a foodborne pathogen? World J Gastroenterol. 2018;24(31):3472-87. https://doi.org/10.3748/wjg.v24.31.3472.

44. Thomson CA, Stanaway JD, Neuhouser ML, Snetselaar LG, Stefanick ML, Arendell $\mathrm{L}$, et al. Nutrient intake and anemia risk in the women's health initiative observational study. J Am Diet Assoc. 2011;111(4):532-41. https:// doi.org/10.1016/j.jada.2011.01.017.

45. Wittenbecher C, Muhlenbruch K, Kroger J, Jacobs S, Kuxhaus O, Floegel A, et al. Amino acids, lipid metabolites, and ferritin as potential mediators linking red meat consumption to type 2 diabetes. Am J Clin Nutr. 2015; 101(6):1241-50. https://doi.org/10.3945/ajcn.114.099150.

46. Cade JE, Moreton JA, O'Hara B, Greenwood DC, Moor J, Burley VJ, et al. Diet and genetic factors associated with iron status in middle-aged women. Am J Clin Nutr. 2005;82(4):813-20. https://doi.org/10.1093/ajcn/82.4.813.

47. Galan P, Yoon HC, Preziosi P, Viteri F, Valeix P, Fieux B, et al. Determining factors in the iron status of adult women in the SU.VI.MAX study. SUpplementation en VItamines et Mineraux AntioXydants. Eur J Clin Nutr. 1998;52(6):383-8. https://doi.org/10.1038/sj.ejcn.1600561.

48. Rigas AS, Sorensen CJ, Pedersen OB, Petersen MS, Thorner LW, Kotze S, et al. Predictors of iron levels in 14,737 Danish blood donors: results from the Danish Blood Donor Study. Transfusion. 2014;54(3 Pt 2):789-96. https://doi. org/10.1111/trf.12518.

49. Tong TYN, Key TJ, Gaitskell K, Green TJ, Guo W, Sanders TA, et al. Hematological parameters and prevalence of anemia in white and British Indian vegetarians and nonvegetarians in the UK Biobank. Am J Clin Nutr. 2019. https://doi.org/10.1093/ajcn/nqz072.

50. Cook JD. Adaptation in iron metabolism. Am J Clin Nutr. 1990;51(2):301-8. https://doi.org/10.1093/ajcn/51.2.301.

51. Aune $D$, Ursin $G$, Veierod MB. Meat consumption and the risk of type 2 diabetes: a systematic review and meta-analysis of cohort studies. Diabetologia. 2009;52(11):2277-87. https:/doi.org/10.1007/s00125-009-1481-X.

52. Ericson U, Hindy G, Drake I, Schulz CA, Brunkwall L, Hellstrand S, et al. Dietary and genetic risk scores and incidence of type 2 diabetes. Genes Nutr. 2018:13:13. https://doi.org/10.1186/s12263-018-0599-1.

53. Kurotani K, Nanri A, Goto A, Mizoue T, Noda M, Oba S, et al. Red meat consumption is associated with the risk of type 2 diabetes in men but not in women: a Japan Public Health Center-based Prospective Study. Br I Nutr. 2013;110(10):1910-8. https://doi.org/10.1017/s0007114513001128.

54. Bao W, Rong Y, Rong S, Liu L. Dietary iron intake, body iron stores, and the risk of type 2 diabetes: a systematic review and meta-analysis. BMC Med. 2012;10:119. https://doi.org/10.1186/1741-7015-10-119.

55. Kim Y, Keogh J, Clifton P. A review of potential metabolic etiologies of the observed association between red meat consumption and development of type 2 diabetes mellitus. Metab Clin Exp. 2015;64(7):768-79. https://doi.org/ 10.1016/.j.metabol.2015.03.008.

56. Vergnaud AC, Norat T, Romaguera D, Mouw T, May AM, Travier N, et al. Meat consumption and prospective weight change in participants of the EPIC-PANACEA study. Am J Clin Nutr. 2010;92(2):398-407. https://doi.org/10. 3945/ajcn.2009.28713.

57. Mozaffarian D, Hao T, Rimm EB, Willett WC, Hu FB. Changes in diet and lifestyle and long-term weight gain in women and men. N Engl J Med. 2011;364(25):2392-404. https://doi.org/10.1056/NEJMoa1014296.

\section{Publisher's Note}

Springer Nature remains neutral with regard to jurisdictional claims in published maps and institutional affiliations.

Ready to submit your research? Choose BMC and benefit from:
- fast, convenient online submission
- thorough peer review by experienced researchers in your field
- rapid publication on acceptance
- support for research data, including large and complex data types
- gold Open Access which fosters wider collaboration and increased citations
- maximum visibility for your research: over 100M website views per year
At BMC, research is always in progress.
Learn more biomedcentral.com/submissions

